Current Drections in

Psychological

SCIENCE

\title{
A Neural Basis for Phonological Awareness? An Oscillatory "Temporal Sampling" Perspective
}

\begin{tabular}{|c|c|}
\hline Journal: & Current Directions in Psychological Science \\
\hline Manuscript ID & CDPS-16-0172.R1 \\
\hline Manuscript Type: & Invited Manuscript \\
\hline Date Submitted by the Author: & $\mathrm{n} / \mathrm{a}$ \\
\hline Complete List of Authors: & Goswami, Usha; University of Cambridge, Psychology \\
\hline Keywords: & dyslexia, phonology, rhythm, oscillations \\
\hline Abstract: & $\begin{array}{l}\text { Individual differences in 'phonological awareness' or speech sound } \\
\text { awareness between children predict reading and spelling development } \\
\text { across languages, and children with dyslexia have impaired phonological } \\
\text { awareness. Recent advances in our understanding of the neural basis of } \\
\text { speech encoding suggest one possible sensory/neural basis for these } \\
\text { individual differences. An oscillatory theoretical perspective is described, } \\
\text { based on sampling of the speech stream by networks of cells that vary in } \\
\text { excitability at different temporal rates. These variations in neural } \\
\text { excitability (oscillations) may align to similar energy variations (such as } \\
\text { amplitude modulations, AMs) in speech, helping to encode the signal. } \\
\text { Indeed, cell networks in auditory cortex form an oscillatory hierarchy, } \\
\text { which mirrors an AM hierarchy found in rhythmic speech. Mappings } \\
\text { between these hierarchies may support parsing of the speech signal into } \\
\text { phonological units. Oscillations at } 2 \text { Hz may help identify stressed } \\
\text { syllables, used to convey meaning in all languages, while oscillations at } ~ \\
\text { Hz may help identify syllables. Behavioural research suggests that the } \\
\text { rhythmic patterning of stressed syllables may provide an acoustic 'skeleton' } \\
\text { or scaffold for phonological development across languages. As well helping } \\
\text { to explain individual differences, an oscillatory framework offers new } \\
\text { targets for improving children's phonological development, for example via } \\
\text { multi-modal rhythmic activities. }\end{array}$ \\
\hline
\end{tabular}

\section{SCHOLARONE \\ Manuscripts}




\begin{abstract}
A Neural Basis for Phonological Awareness?
An Oscillatory “Temporal Sampling” Perspective
\end{abstract}

\section{Usha Goswami}

Centre for Neuroscience in Education, Department of Psychology University of Cambridge, Downing St, Cambridge CB2 3EB, U.K.

Running Head: Neural basis of phonological awareness

\begin{abstract}
Corresponding Author:
\end{abstract}
Professor Usha Goswami

Department of Psychology

Downing St., Cambridge CB2 3EB, U.K.

Tel. 441223767635 Fax. 441223333564

Email: ucg10@cam.ac.uk 


\begin{abstract}
Individual differences in 'phonological awareness' or speech sound awareness between children predict reading and spelling development across languages, and children with dyslexia have impaired phonological awareness. Recent advances in our understanding of the neural basis of speech encoding suggest one possible sensory/neural basis for these individual differences. An oscillatory theoretical perspective is described, based on sampling of the speech stream by networks of cells that vary in excitability at different temporal rates. These variations in neural excitability (oscillations) may align to similar energy variations (such as amplitude modulations, AMs) in speech, helping to encode the signal. Indeed, cell networks in auditory cortex form an oscillatory hierarchy, which mirrors an AM hierarchy found in rhythmic speech. Mappings between these hierarchies may support parsing of the speech signal into phonological units. Oscillations at $\sim 2 \mathrm{~Hz}$ may help identify stressed syllables, used to convey meaning in all languages, while oscillations at $\sim 5 \mathrm{~Hz}$ may help identify syllables. Behavioural research suggests that the rhythmic patterning of stressed syllables may provide an acoustic 'skeleton' or scaffold for phonological development across languages. As well as helping to explain individual differences, an oscillatory framework offers new targets for improving children's phonological development, for example via multimodal rhythmic activities.
\end{abstract}

Keywords: dyslexia, oscillations, phonology, rhythm 


\section{Introduction}

Children's phonological awareness is an important causal factor in their reading and spelling development, across languages (Ziegler \& Goswami, 2005). Phonological awareness in young children is typically measured using oral tasks, for example requiring rhyme judgements ("cat" "fit" "hat"), syllable counting ("hospital" has 3 syllables), judgements about syllable stress patterns (ma-TER-nity versus MA-ternity), and the deletion of individual speech sounds from words ( "star" without "s" leaves "tar"). Phonological awareness in children appears to develop in an hierarchical fashion. Pre-reading children and illiterate adults across languages are aware of phonological units at larger 'grain sizes' - stressed syllables, syllables and rhymes. As the brain learns to read, phonological awareness tasks based on the individual speech sounds reflected by the alphabet are performed successfully ('phoneme awareness'). Although many models of reading development and dyslexia consider phonemic awareness to be a key causal factor (e.g., Hulme \& Snowling, 2013), difficulties with phonemes may well reflect earlier difficulties with phonology at larger grain sizes (e.g., Goswami, 2015). While phonology is considered a cognitive system, phonological awareness most likely develops initially on the basis of sensory information in the speech signal. The nature of this sensory information has been hotly debated (Goswami, 2015, for a recent review).

Work with infants has revealed a range of sensory cues that support phonological learning, including exquisite statistical sensitivity to transitional probabilities between syllables (Saffran, 2001) and fine-grained phonetic learning effects such as distinguishing native from non-native phonemes (Kuhl, 2004). The importance of these types of statistical learning in early language acquisition is now understood to be fundamental. However, neural research on speech encoding by adults reveals another potential source of statistical learning about speech. The rhythmic energy patterns (amplitude modulations, AMs) in the speech 
envelope also provide important statistical information. The infant brain is sensitive to these rhythmic energy patterns from birth (Telkemyer et al., 2011), however individual differences in sensitivity to AM patterning in infancy have yet to be explored. Meanwhile, research with children with dyslexia suggests that the accuracy with which these AM patterns are encoded by the brain might be critical for the development of an efficient phonological system (“Temporal Sampling" theory, Goswami, 2011).

\section{Amplitude Modulation, the Amplitude Envelope of Speech and Amplitude "Rise Times"}

The acoustic speech signal is very complex, and it can be modelled in various ways, the most familiar being the speech spectrogram. The amplitude envelope of speech is not visible in the spectrogram. The amplitude envelope is the energy profile of the raw acoustic wave form (see Figure 1). This energy profile varies relatively slowly in time, reflecting the fact that most of the power fluctuations reflect the energy "arcs" that coincide with syllable production (Greenberg, 2006). Syllable by syllable, peak energy is reached as each vowel as produced, and then falls again. The rising phase of each energy arc corresponds to syllable "rise time". Perceptually, we experience these energy fluctuations in the envelope as speech rhythm. Sensitivity to these AM-driven rhythms is important for speech intelligibility. The brain can recognise speech on the basis of the amplitude and temporal cues in the envelope alone (Shannon et al. 1995). In such studies, the temporal fine structure that is the focus of much current infant research has been removed. Such studies suggest that the AM patterns in speech may provide additional important statistical information about language, information relevant to phonological development. 
(a) Example of Speech Amplitude Envelope

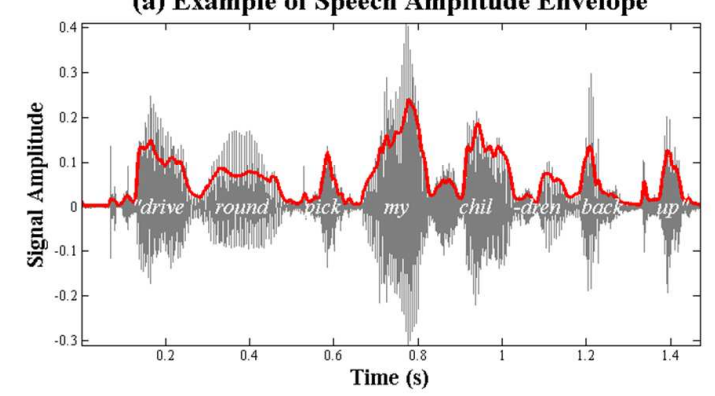

(b) Onset Rise Time for "my"

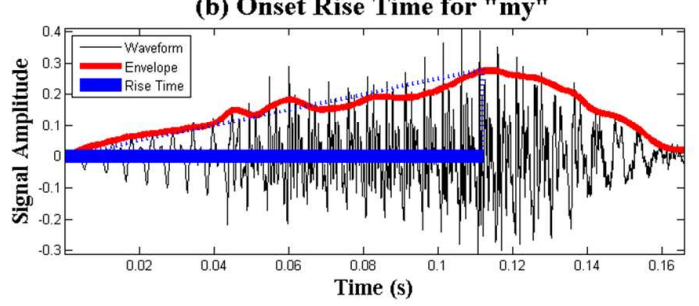

Figure 1. Panel (a) shows the energy profile of the sentence fragment “..drive around, pick my children back up.." plotted as signal amplitude against time. The amplitude envelope is shown in red. The rise time cues associated with individual syllables are clearly visible; nevertheless not every rise in amplitude corresponds to a syllable onset. Panel (b) shows the syllable "my" in greater detail, with the rise time plotted. As shown, the rise time does not equate to a single moment in time, and the time taken for the envelope to reach its peak amplitude will vary syllable by syllable. Figure adapted from Goswami \& Leong, 2013; Laboratory Phonology, 4, 67-92.

Indeed, research with children who have poor phonological awareness (children with developmental dyslexia) reveals a difficulty in discriminating the "rise times" of non-speech amplitude envelopes, across languages (e.g., English, Spanish, Chinese, Dutch, Finnish, French and Hungarian; see Goswami, 2015). Rise time discrimination in these studies is typically associated with individual differences in phonological awareness of languagerelevant phonological units (e.g., tone awareness in Chinese, phoneme awareness in Spanish, rhyme awareness in English, see Goswami et al. 2011), and with individual differences in reading development (including in non-alphabetic orthographies like Chinese, Wang et al., 2012). This behavioural difficulty in rise time discrimination suggests a sensory difficulty in identifying different AM rates. There are multiple quasi-rhythmic energy fluctations at both faster and slower rates nested in the amplitude envelope of speech, each identified by their "rise times". The overall amplitude envelope is a power-weighted average of these different fluctuations. Temporal Sampling theory proposed that the slower rates of energy variation $<10 \mathrm{~Hz}$, which govern rhythm perception, are the main source of dyslexic children's 
perceptual difficulties. On this hypothesis, the accurate perception of slower AMs would be important for the development of phonological awareness by all children.

The AM patterns nested in the envelope of child-directed speech can be identified by analysing the temporal modulation structure of English nursery rhymes (Leong \& Goswami, 2015; see Figure 2). English nursery rhymes typically have simple metrical structures, with clear rhythmic patterns based on the alternation of strong ( $\mathrm{S}$, louder) and weak (W, softer) syllables (for example, "Jack and Jill went up the hill” follows a trochaic rhythm pattern, SWSWSWS). In adult-directed speech, rise times occurring around 5 times a second (a rate of $\sim 5 \mathrm{~Hz}$ ) appear dominant, relating to the production of syllables (strong and weak;

Greenberg et al., 2003). In nursery rhymes and also in infant-directed speech, however, prominent rise times occur twice a second $(2 \mathrm{~Hz})$, relating to the production of stressed syllables. This can be seen in Figure 2. Consistent with the sensory rise time studies, brain imaging studies of children with dyslexia suggest that individual differences in children's encoding of these $\sim 2 \mathrm{~Hz}$ modulations plays an important role in individual differences in phonological learning.

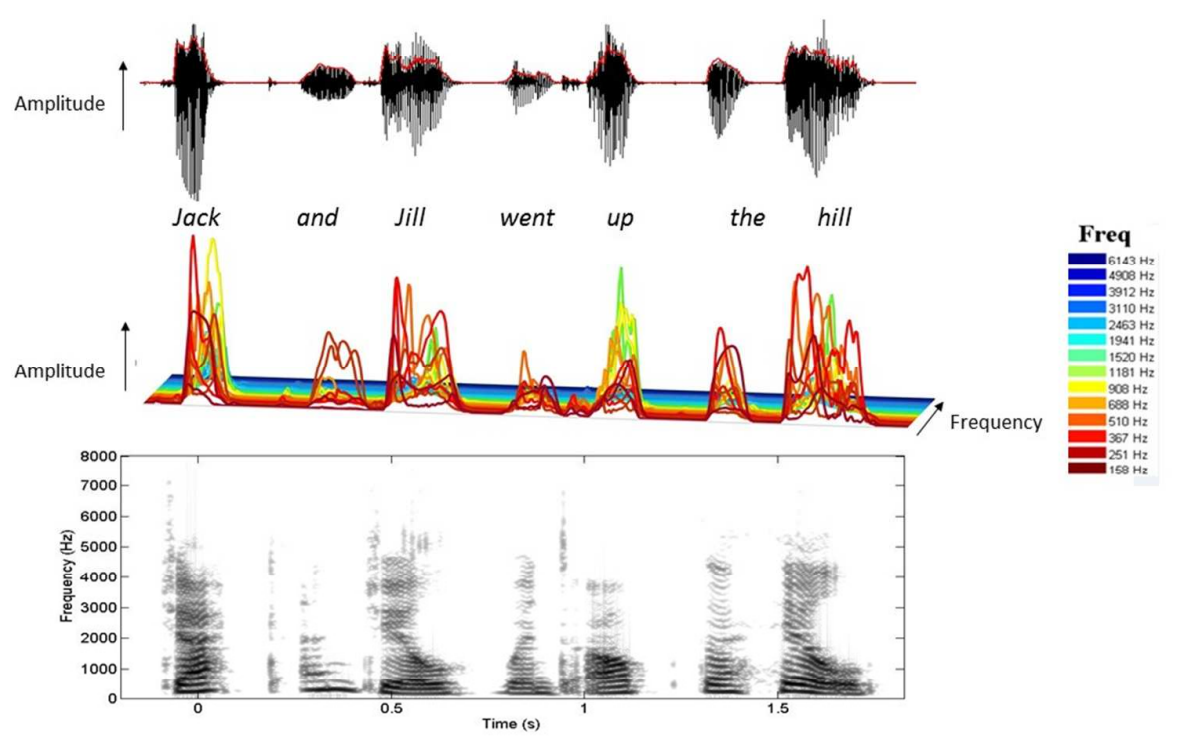


Figure 2. The top panel shows the energy profile of the raw speech signal over time (seconds) for the nursery rhyme "Jack and Jill went up the hill" with the amplitude envelope (power-weighted average) plotted in red. The middle panel unpacks the averaged envelope to show the amplitude envelopes for different spectral frequency bands, colour-coded from low frequencies (red colours, foreground) to high frequencies (blue colours, background). The energy fluctuations that correspond to the stressed syllables like "Jack" and "hill" are very salient, particularly in the lower spectral frequency bands in the speech signal. The bottom panel shows the same speech information plotted as a speech spectrogram. The salient amplitude changes in the lower frequencies are here depicted via increased shading, a pictorial choice which makes their possible salience for the brain less obvious. Figure by Sheila Flanagan.

\section{How Does the Brain Encode Modulation Patterns?}

Brain cells (neurons) have a spontaneous quasi-rhythmic firing rate even when the brain is at rest, as they are either in an excitatory state (sending an electrical pulse) or recovering (inhibitory state). Neurons are arranged in large networks in the brain, and in the auditory cortex these networks have preferred oscillatory rates that are thought to be important for speech encoding (Giraud \& Poeppel, 2012). For example, amplitude rise times in the theta band in speech $(4-8 \mathrm{~Hz}$, so $4-8$ fluctuations per second) are important for resetting the quasi-rhythmic activity of neural networks so that they are "in sync" (or in phase) with these energy variations in speech (Luo \& Poeppel, 2007). This process is called oscillatory phase alignment or phase entrainment. During phase entrainment, cell networks recalibrate their spontaneous activity so that it is aligned temporally with energy modulations in speech. If phase entrainment is accurate, then peaks and troughs in speech energy are faithfully tracked by peaks and troughs in electrical activity (see Doelling et al., 2014). Indeed, Doelling and colleagues showed that for adult listeners, removing syllable-rate rise time markers (theta rate landmarks, here $2-9 \mathrm{~Hz}$ ), rendered speech unintelligible. Oscillatory phase entrainment to speech in children can be studied using electrophysiology and the electroencephalogram (EEG). By recording variations in the brain's electrical energy directly (using sensors on the scalp), EEG can reveal the phase alignment of cell network 
activity with energy variations in the speech signal. For speech, important cell signalling rates are delta $(1-3 \mathrm{~Hz})$, theta $(4-8 \mathrm{~Hz})$, beta $(15-30 \mathrm{~Hz})$ and gamma (> $30 \mathrm{~Hz}$, Poeppel, 2014).

In our EEG studies, children with dyslexia show a significant difference in preferred phase in the delta band when listening to rhythmic speech compared to control children, but not in the theta band (Power et al., 2013). Preferred phase refers to the timepoint in a temporal cycle when most neurons discharge their electrical pulses. So for children with dyslexia, neural firing rhythms were less well-aligned with speech rhythms at slower rates (delta). In other studies, we used the electrical brain signal to resynthesise the acoustic patterns in the input signal (the speech envelopes of sentences), thereby indexing the neural quality of children's phonological representations. We found that speech envelopes in a $0-2$ $\mathrm{Hz}$ (delta) band were encoded less accurately by the children with dyslexia (Power et al., 2016), even when the children with dyslexia were compared to reading-level matched children who were 2 years younger. This suggests a fundamental encoding deficit, which other studies suggest persists into adulthood (Soltész et al., 2013; Molinaro et al., 2016). In the EEG studies, individual differences in the different neural measures were significantly related to children's phonological development.

Atypical neural encoding of slower speech rhythm information from birth would be expected to impair the trajectory of language development in affected children. Indeed, rise time discrimination is also impaired in children with overt speech and language difficulties, who also show impairments in perceiving speech rhythm (e.g., Cumming et al., 2015). Children with overt speech and language impairments, unlike children with dyslexia, may be impaired in processing faster temporal modulation patterns in speech (low gamma rate, see Goswami et al., 2016). Meanwhile, impaired rise time discrimination is already found in English-exposed infants who are at family (genetic) risk for dyslexia (Kalashnikova et al., 2016) as well as in Dutch-speaking pre-schoolers at family risk for dyslexia (Law et al., 
2016). If similar data are found in other languages, then a Temporal Sampling framework may help to explain the impairments in phonological awareness that are found across languages in children with dyslexia, including non-Indo-European languages. Infants across languages appear to begin parsing speech on the basis of rhythm and stress, and some linguists propose that all languages can be arranged on a continuum of more-or-less stress based (Dauer, 1983). Accordingly, atypical sensory/neural processing of linguistic stress patterns would have consequences for phonological development in all languages.

\section{Phonological Development and Rhythmic Synchronisation Across Modalities}

Analyses of oscillatory phase (these cyclical patterns) are an important way of quantifying rhythmic behaviour. Phase analyses can also be applied to the temporal alignment of non-neural behaviours with a rhythmic timekeeper, for example finger tapping to a beat (beat synchronisation). If basic sensory components of rhythm perception are impaired in dyslexia, such as rise time discrimination, then performance with any beat-based rhythm should also be affected, across modalities. For English-speaking children with dyslexia, we indeed find impairments in both tapping along to a metronome beat (at speeds of $2 \mathrm{~Hz}$ or $2.5 \mathrm{~Hz}$; Thomson \& Goswami, 2008) and detecting whether the rhythm of patterns of musical beats are the same or different (Huss et al., 2011). In these and other developmental studies, individual differences in both tapping accuracy and musical rhythm judgements were unique predictors of individual differences in phonological awareness (see also Delatolas et al., 2009). We have also studied children's sensitivity to the 'beat structure' (SWSW syllable patterning) of words directly using a "dee dee" task (in which familiar phrases are spoken using the syllables "dee" [W] or "DEE" [S], so 'Harry Potter' is DEEdeeDEEdee), and a syllable mis-stressing judgement task (e.g., "difficulty" is spoken with primary stress on the [weak] second syllable, this WSWW pattern is incorrect). Again, children with dyslexia perform poorly in each task, and individual differences are related to rise time sensitivity. 

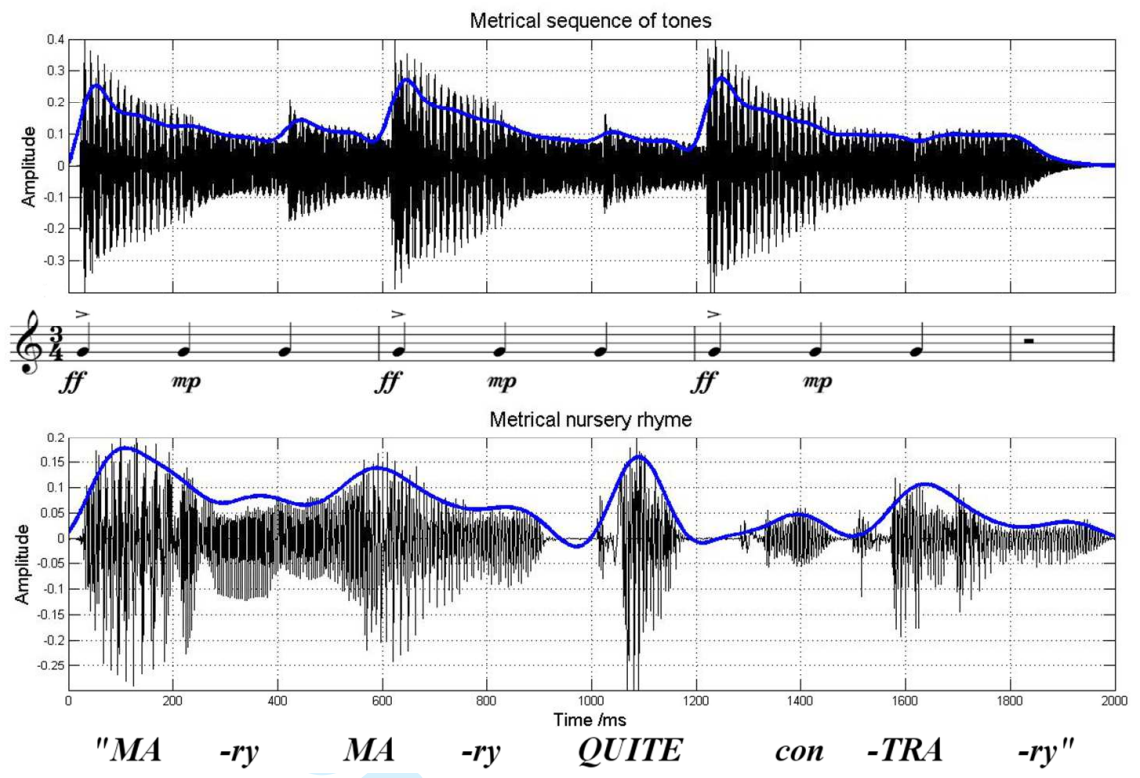

Figure 3. The top panel shows the amplitude envelope for one of the chime bar "tunes" used by Huss et al. (2011), with the amplitude envelope in blue. The bottom panel shows the speech wave form for the nursery rhyme "Mary Mary quite contrary", with the amplitude envelope in blue. According to Temporal Sampling theory, if the amplitude envelopes in musical rhythms are matched with speech (e.g., during singing a nursery rhyme to musical accompaniment), then the accuracy of oscillatory entrainment to speech may be enhanced. Figure from Goswami (2012), Empirical Musicology Review, 7, 1-2, 57-63.

The amplitude envelope of a simple chime bar "tune" used in Huss et al.'s music task is shown in Figure 3, reflecting strong/weak beat patterning. When a note is accented (strong beat), the rise time cue is more salient. As rhythm is more overt in music than in speech, it may be possible to enhance children's sensory perception of speech rhythms using musical rhythms, via aligning the amplitude envelopes. We have designed an intervention for poor readers based on this idea using music and poetry (e.g., bongo drumming to different beat structures in music, clapping out the syllable structure of poems, marching to the beat patterns in nursery rhymes; Bhide et al., 2013). Following Temporal Sampling theory, the activities were based on a $2 \mathrm{~Hz}$ beat rate. Our multi-modal rhythmic intervention had significant effects on the participating children's phonological skills as well as their reading (see also Degé \& Schwarzer, 2011). When we measured tapping accuracy in these children, we found that the degree of improvement in children's beat synchronisation was significantly 
related to their improvement in reading (Bhide et al., 2013). Accordingly, individual differences in tapping to a $2 \mathrm{~Hz}$ beat could be a useful proxy measure of the accuracy of neural rhythmic entrainment, as well as simple to administer in the classroom.

\section{The Oscillatory Hierarchy and the Phonological Hierarchy}

According to Temporal Sampling theory, the reason that practice in beat synchronisation enhances language processing is because multi-modal rhythmic activities "entrain the oscillators", improving the phase alignment of delta- and theta-rate oscillatory networks with the AM patterns in speech (see Goswami \& Szücs, 2011). On this perspective, a cultural activity like nursery rhymes would be analogous to 'supernormal' stimuli in ethology, here acting as supernormal entraining stimuli to promote phonological development. This idea has yet to be investigated with brain imaging. However, we have modelled the temporal modulation structure of children's nursery rhymes (Leong \& Goswami, 2015). The modelling revealed an hierarchy of amplitude modulations in 3 temporal rate bands centred on $\sim 2 \mathrm{~Hz}$ (delta band), $\sim 5 \mathrm{~Hz}$ (theta band) and $\sim 20 \mathrm{~Hz}$ (beta band). The AM hierarchy was governed by the slowest (delta-rate) AMs. Further, cycles of AM at each rate provided an acoustic guide to phonological units (Figure 4). AM cycles centred around $2 \mathrm{~Hz}$ supported the parsing of stressed syllables, AM cycles centred around 5 $\mathrm{Hz}$ supported the parsing of syllables, and AM cycles centred around $20 \mathrm{~Hz}$ supported the parsing of onset-rime units (to divide any syllable into onset and rime, we divide at the vowel, as in $d$-ash, sm-ash, spl-ash). Indeed, the acoustic-phonological mappings achieved by the model when the nursery rhymes were produced to an external (metronome) beat were 95\% accurate for prosodic stress patterns, $98 \%$ accurate for syllables, and $91 \%$ accurate for onset-rime units. Acoustic-phonological mappings were lower for naturally-produced nursery rhymes (i.e., not externally timed), at $72 \%$ correct for prosodic stress patterns, $82 \%$ correct for syllables, and $78 \%$ correct for onset-rime units. The performance of the model suggests 
that the acoustic structure of the amplitude envelope carries significant statistical information that may support phonological development. Experimental studies with infants and children are now required to see whether this "Amplitude Modulation Phase Hierarchy" approach to the statistical modelling of child-directed and infant-directed speech is useful across languages.

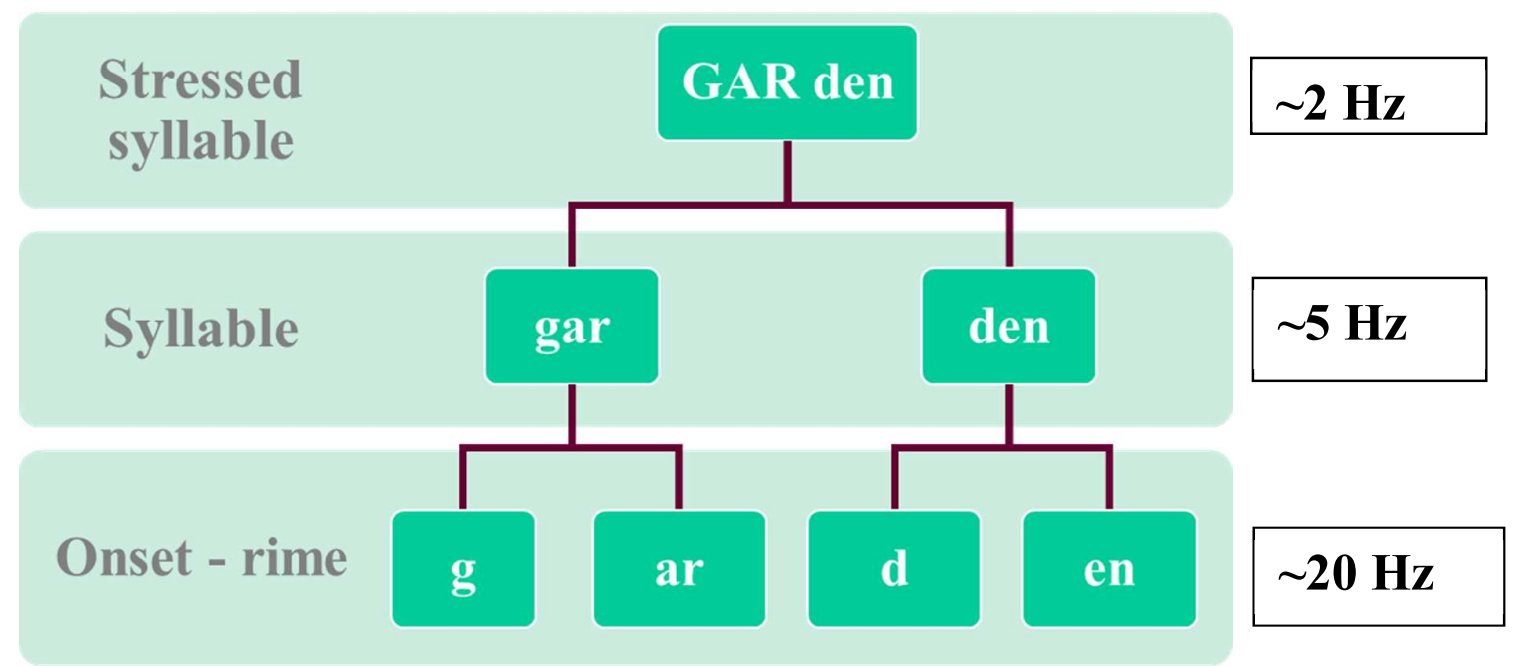

Figure 4. The phonological hierarchy is shown for the bisyllabic word "garden", which has the classic strong-weak syllable pattern that characterises English nouns. The central frequencies for the bands of amplitude modulation in speech computed by the Amplitude Modulation Phase Hierarchy modelling (Leong \& Goswami , 2015) are shown on the right. By hypothesis, children's sensitivity to these AM patterns and the accuracy of automatic cortical oscillatory entrainment in these temporal bands supports parsing of the speech signal into linguistic units. Accordingly, individual differences in the accuracy of neural entrainment to these AM bands and/or sensory discrimination of rise times could be associated with individual differences in children's phonological development.

\section{Conclusion}

A “Temporal Sampling” framework for phonological development offers a sensory/neural mechanistic framework for the development of phonological knowledge by children, which should apply across languages. Encoding of relational structures in the sensory input (the amplitude modulation hierarchy in the speech signal) via specific physiological mechanisms (neuronal oscillations in auditory cortex) that conserve temporal 
relational structure (the oscillatory hierarchy) may support the cognitive extraction of phonology, also an hierarchical relational structure (the linguistic hierarchy of stress feet, syllables, onset-rimes and phonemes, see Goswami, 2015). As infants in all languages begin parsing the speech signal by using stress and rhythm patterns, a Temporal Sampling framework offers a sensory/neural/cognitive theoretical basis for understanding phonological development and phonological impairments across languages. Although not yet tested directly, it is likely that similar temporal modulation hierarchies are present also in the visual and motor encoding of speech (Ghazanfar \& Takahashi, 2014). Accordingly, this theoretical approach has the potential to fill some important gaps in our current understanding of children's language development, for example, concerning the underlying causes of dyspraxia. 


\author{
Bibliography
}

Bhide, A., Power, A.J., and Goswami, U. (2013). A rhythmic musical intervention for poor readers: A comparison of efficacy with a letter-based intervention. Mind Brain \& Education, 7 (2), 113-123.

Cumming, R., Wilson, A., and Goswami, U. (2015). Basic auditory processing and sensitivity to prosodic structure in children with specific language impairments: A new look at a perceptual hypothesis. Frontiers in Psychology, 6, 972.

Dauer, R.M. (1983) Stress-timing and syllable-timing re-analysed, Journal of Phonetics, 11, 51-62.

Degé, F., and Schwarzer, G. (2011). The effect of a music program on phonological awareness in preschoolers. Frontiers in Psychology, 2, 124.

Dellatolas, G., Watier, L., Le Normand, M-T., Lubart, T., and Chevrie-Muller, C. (2009). Rhythm reproduction in kindergarten, reading performance at second grade, and developmental dyslexia theories. Archives of Clinical Neuropsychology, 24, 555-563.

Doelling, K.B., Arnal, L.H., Ghitza, O., and Poeppel, D. (2014) Acoustic landmarks drive delta-theta oscillations to enable speech comprehension by facilitating perceptual parsing. Neuroimage. 85, 761-768.

Giraud, A.L., and Poeppel, D. (2012).Cortical oscillations and speech processing: emerging computational principles and operations. Nature Neuroscience, 15, 511-517.

Ghazanfar, A.A., and Takahashi, D.Y. (2014). The evolution of speech: vision, rhythm, cooperation. Trends in Cognitive Science, 18, 543-553.

Goswami, U. (2011). A temporal sampling framework for developmental dyslexia. Trends in Cognitive Science, 15, 3-10.

Goswami, U. (2012). Entraining the brain: Applications to language research and links to musical entrainment. Empirical Musicology Review, 7(1-2), 57-63.

Goswami, U. (2015). Sensory theories of developmental dyslexia: three challenges for research. Nature Reviews Neuroscience, 16(1), 43-54.

Goswami, U., Cumming, R., Chait, M., Huss, M., Mead, N., Barnes, L., Wilson, A., and Fosker, T. (2016). Perception of filtered speech by children with developmental dyslexia and children with specific language impairments. Frontiers in Psychology, 7, 791.

Goswami, U., and Leong, L. (2013). Speech rhythm and temporal structure: Converging perspectives? Laboratory Phonology, 4(1), 67-92.

Goswami, U., and Szücs, D. (2011). Educational Neuroscience: Developmental Mechanisms; Towards a conceptual framework. Neuroimage, 57, 651-8. 
Goswami, U., Wang, H-L., Cruz, A., Fosker, T., Mead, N., and Huss, M. (2011). Languageuniversal sensory deficits in developmenta dyslexia: English, Spanish and Chinese. Journal of Cognitive Neuroscience, 23, 325-337.

Greenberg, S. (2006). A multi-tier framework for understanding spoken language. In S Greenberg and W Ainsworth (Eds.), Listening to Speech - An Auditory Perspective, pp. 411-433. Hillsdale, NJ: Lawrence Erlbaum Associates.

Greenberg, S., Carvey, H., Hitchcock, L., and Chang, S. (2003). Temporal properties of spontaneous speech - a syllable-centric perspective. Journal of Phonetics, 31, 465485.

Hulme, C, and Snowling, M. (2013). Learning to Read: What we know and what we need to understand better. Child Development Perspectives, 7 (1), 1-5.

Huss, M., Verney, J.P., Fosker, T., Mead, N., and Goswami, U. (2011). Music, rhythm, rise time perception and developmental dyslexia: Perception of musical meter predicts reading and phonology. Cortex, 47, 674-89.

Kalashnikova, M., Goswami, U., and Burnham, D. (2016). Mothers speak differently to infants at risk for dyslexia. Developmental Science, DOI: 10.1111/desc. 12487.

Kuhl, P.K. (2004). Early language acquisition: Cracking the speech code. Nature Reviews Neuroscience, 5, 831-843.

Law, J.M., Wouters, J., and Ghesquiere, P. (2016). The influences and outcomes of phonological awareness: A study of MA, PA and auditory processing in pre-schoolers with a family risk of dyslexia. Developmental Science, DOI: 10.1111/desc. 12453.

Leong, V., and Goswami, U. (2015). Acoustic-emergent phonology in the amplitude envelope of child-directed speech. PLoS One, 10 (12), e0144411.

Luo, H., and Poeppel, D. (2007). Phase patterns of neuronal responses reliably discriminate speech in human auditory cortex. Neuron, 54, 1001-10.

Molinaro N, Lizarazu M, Lallier M, Bourguignon M, Carreiras M. (2016). Out-of-synchrony speech entrainment in developmental dyslexia. Human Brain Mapping, 37, 27672783.

Poeppel, D. (2014). The neuroanatomic and neurophysiological infrastructure for speech and language. Current Opinion in Neurobiology, 28, 142-149.

Power, A.J., Mead, N., Barnes, L. and Goswami, U. (2013). Neural entrainment to rhythmic speech in children with developmental dyslexia. Frontiers in Human Neuroscience, 7, 777.

Power, A.J., Colling, L.C., Mead, N., Barnes, L., and Goswami, U. (2016). Neural encoding of the speech envelope by children with developmental dyslexia. Brain \& Language, $160,1-10$.

Saffran, J.R. (2001). Words in a sea of sounds: The output of infant statistical learning. Cognition, 81, 149-169. 
Shannon, R.V., Zeng, F-G, Kamath, V., Wygonski, J., and Ekelid, M. (1995). Speech recognition with primarily temporal cues. Science, 270, 303-304.

Soltész, F., Szücs, D., Leong, V., White, S. and Goswami, U. (2013) Differential Entrainment of Neuroelectric Delta Oscillations in Developmental Dyslexia. PLoS ONE 8(10), e76608.

Telkemeyer S, Rossi S, Nierhaus T, Steinbrink J, Obrig H, and Wartenburger I. (2011). Acoustic processing of temporally-modulated sounds in infants: Evidence from a combined NIRS and EEG study. Frontiers in Psychology, 2, 62.

Thomson, J., and Goswami, U. (2008). Rhythmic processing in children with developmental dyslexia: Auditory and motor rhythms link to reading and spelling. Journal of Physiology Paris, 102, 120-129.

Wang, H.L.S., Huss, M., Hämäläinen, J., and Goswami, U. (2012). Basic auditory processing and developmental dyslexia in Chinese. Reading \& Writing, 25 (2), 509-536.

Ziegler, J.C., and Goswami, U. (2005). Reading acquisition, developmental dyslexia, and skilled reading across languages: A psycholinguistic grain size theory. Psychological Bulletin, 131, 3-29. 